\title{
Toward Baselining Software Anomalies in NASA Missions
}

\author{
Lucas Layman $^{1}$, Marvin Zelkowitz ${ }^{12}$, Victor Basili ${ }^{123}$ \\ ${ }^{1}$ Fraunhofer Center for Experimental Software Eng. \\ ${ }^{2}$ University of Maryland, College Park \\ ${ }^{3}$ King Abdulaziz University \\ College Park, MD 20740 \\ llayman@fc-md.umd.edu; \{mvz,basili\}@cs.umd.edu
}

\author{
Allen P. Nikora \\ Jet Propulsion Laboratory, \\ California Institute of Technology \\ Pasadena, CA 91109 \\ Allen.P.Nikora@jpl.nasa.gov
}

\begin{abstract}
In this fast abstract, we provide preliminary findings an analysis of 14,500 spacecraft anomalies from unmanned NASA missions. We provide some baselines for the distributions of software vs. non-software anomalies in spaceflight systems, the risk ratings of software anomalies, and the corrective actions associated with software anomalies.
\end{abstract}

Keywords-anomaly; software defect;NASA;baseline

\section{INTRODUCTION}

The importance of software quality, safety, and reliability in spaceflight is increasing. Avionics, guidance, command handling, and fault detection are increasingly pushed into software systems to lighten spacecraft while infusing them with increasingly complex capabilities. In spaceflight systems (as in all software systems), baselines for software quality and reliability are needed to establish if system behavior falls within acceptable performance parameters, and to help guide development efforts intent on meeting software quality and reliability requirements.

An integral component to creating baselines for software quality and reliability are post-release failures. At NASA, incident surprise anomalies (ISAs) are erroneous or unusual observed behaviors from spacecraft (e.g. satellites, planetary explorers) that are reported by mission operations teams. These anomalies typically indicate an error in the spacecraft operation due to a component failure (hardware or software on the spacecraft or ground) or a procedural error by the operations team. These anomalies are recorded in anomaly databases, triaged, and addressed by the project team. We examined the anomaly databases from the NASA Jet Propulsion Lab (JPL) and Goddard Space Flight Center (GSFC), which contain over 39,000 anomalies. We analyzed a subset of 14,500 anomalies to baseline the number of software-related anomalies and to characterize them according to their corrective actions and risk ratings (mission impact and severity).

\section{ANOMALY DATASET DESCRIPTION}

The missions selected for analysis are based on the quality of the anomaly information available for that project in the database. Anomalies recorded prior to 1989 for JPL and prior to 1997 for GSFC were recorded either on paper or in legacy systems, often resulting in too much missing information for meaningful analysis. A summary of the datasets is provided in Table 1.

TABLE 1. ANOMALY DATA SET SUMMARY

\begin{tabular}{|c|c|c|c|c|}
\hline Center & $\begin{array}{c}\text { Total } \\
\text { anomalies }^{\text {a }}\end{array}$ & $\begin{array}{c}\text { Subset } \\
\text { analyzed }\end{array}$ & $\begin{array}{c}\# \\
\text { Missions }\end{array}$ & $\begin{array}{c}\text { Launch } \\
\text { dates }\end{array}$ \\
\hline JPL & 13696 & 4621 & 9 & $1989-2005$ \\
\hline GSFC & 25320 & 9921 & 29 & $1997-2010$ \\
\hline
\end{tabular}

a. These numbers represent only the anomalies exposed to us in the current anomaly databases.

Each anomaly is indexed by a unique anomaly number for its database. The anomaly reports contain descriptions of the anomalous event, a description of the root cause, and a description of the corrective action. The anomalies also contain basic information, including the spacecraft they were observed in, the date the anomaly was observed, and the date the anomaly was closed due to either resolution or inactivity. The anomaly reports also contain fields categorizing the anomaly cause (e.g. software, hardware, unknown), however, these fields are not standardized and sometimes contain missing data. JPL anomaly reports also contain a risk rating (unacceptable risk, accepted risk, no significant risk, no risk), while GSFC anomalies contains a mission impact rating (catastrophic, major, minor, no impact).

\section{SOFTWARE ANOMAly ANALYSIS}

Identifying anomalies that are either caused or corrected by software is not trivial since the categorizations of the anomaly cause in the report were often missing or inaccurate. As such, we created a naïve search algorithm to look for software keywords in the anomaly reports, e.g. software, fsw (flight software), gsw (ground software), s/w, etc. Eliminating the false positives returned by the search algorithm is a manual process. Analysis suggests that the false positive rate for the search algorithm may be as high as $29 \%$ for GSFC and $6 \%$ for JPL. We ignore false negatives for now due to the size of the anomaly databases.

\section{A. How many anomalies are software-related?}

The number of software anomalies found in our analysis subsets is summarized in Table 2. The number of software anomalies is the number of anomalies containing a software search term in the cause description. For both data sets, the causes of approximately $25 \%$ of all anomalies appear to 
involve software. "Software-related anomalies" are anomaly reports where software is part of the corrective action, but not identified as a cause. These software-related anomalies may indicate a hidden software problem; if the software preventing the problem fails, then software may also be considered a cause of the anomaly.

TABLE 2. SOFTWARE ANOMALIES

\begin{tabular}{|c|c|c|c|}
\hline Center & Subset analyzed & \# S/W anom. & \# s/w related anom. \\
\hline JPL & 4621 & 994 & 257 \\
\hline GSFC & 9921 & 2328 & $634^{\mathrm{a}}$ \\
\hline
\end{tabular}

a. Partial estimate - several anomalies still being studied. Actual number probably around 300-400.

\section{B. How are software anomalies corrected?}

We manually inspected the 994 software anomalies from JPL and a random sample of 339 GSFC software anomalies to categorize the corrective action as one of the following:

- $\quad$ software - update, configuration change;

- $\quad$ hardware - replacing a broken ground component;

- $\quad$ operations - actions taken by the mission operations team, such as a procedural workaround or resetting connections;

- $\quad$ none - no action was taken by the mission team;

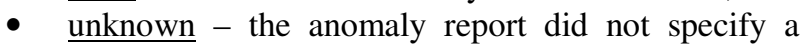
corrective action.

The distributions of the corrective action categories are presented in Table 3.

TABLE 3. SOFTWARE ANOMALY CORRECTIVE ACTIONS

\begin{tabular}{|c|c|c|c|c|c|}
\hline Center & S/W & H/W & Ops & None & Unk. \\
\hline JPL & $577(58 \%)$ & $22(2 \%)$ & $200(20 \%)$ & $116(12 \%)$ & $79(8 \%)$ \\
\hline GSFC & $112(33 \%)$ & 0 & $148(44 \%)$ & $27(8 \%)$ & $52(15 \%)$ \\
\hline
\end{tabular}

These distributions highlight two interesting points. First, in $\sim 10 \%$ of software anomalies, no action is taken when a software anomaly occurs. Either the anomaly was deemed unimportant, or the risks introduced by attempting to correct the problem outweighed the perceived residual risk. Second, operations fixes are common. Often the perception is that, if the operations team can find a workaround either through procedures or in resetting/rebooting the ground software, this is an acceptable if a software patch is perceived as risky.

\section{What is the risk distribution of software anomalies?}

The risk ratings for JPL anomalies (Table 4) indicate the residual risk, while the mission impact ratings for GSFC anomalies (Table 5) indicate the criticality without considering probability. The risk ratings are from the subsets of anomalies analyzed in Table 2.

In the JPL data, software anomalies account for $27 \%$ "Unacceptable" risk anomalies are due to software, and software accounts for $\sim 25 \%$ of all anomalies. In the GSFC data, software anomalies account for only $10 \%$ of "Major" impact anomalies, though software accounts for $\sim 27 \%$ of all anomalies.
TABLE 4. RISK RATING DISTRIBUTIONS FOR JPL ANOMALIES

\begin{tabular}{|c|c|c|c|c|c|}
\hline Risk rating & $\begin{array}{c}\text { Un- } \\
\text { acceptable }\end{array}$ & Accepted & $\begin{array}{c}\text { Not } \\
\text { significant }\end{array}$ & None & Total $^{\mathbf{a}}$ \\
\hline S/W anom. & 44 & 251 & 540 & 45 & 880 \\
\hline$\%$ of S/W & $5.7 \%$ & $34.4 \%$ & $53.7 \%$ & $6.2 \%$ & \\
\hline \hline All anom. & 164 & 995 & 1554 & 179 & 2892 \\
\hline$\%$ of all & $5.00 \%$ & $28.5 \%$ & $61.4 \%$ & $5.1 \%$ & \\
\hline
\end{tabular}

TABLE 5. MISSION IMPACT RATING DISTRIBUTIONS FOR GSFC ANOMALIES

\begin{tabular}{|c|c|c|c|c|c|}
\hline Mission impact & Catastrophic & Major & Minor & None & Total $^{\text {a }}$ \\
\hline S/W anomalies & 0 & 27 & 784 & 679 & 1490 \\
\hline & $0 \%$ & $1.8 \%$ & $52.6 \%$ & $45.6 \%$ & \\
\hline \hline All anomalies & 0 & 261 & 4164 & 2853 & 7278 \\
\hline & $0 \%$ & $3.6 \%$ & $57.2 \%$ & $39.2 \%$ & \\
\hline
\end{tabular}

\section{SUMMARY}

This preliminary analysis raises some interesting points. First, software is the cause of approximately $25 \%$ of all anomalies. Furthermore, software accounts for $26 \%$ of "unacceptable risk" anomalies for JPL and 10\% of "major impact" anomalies for GSFC. Second, the software-related anomalies in Table 2 represent potentially hidden software risk should the software preventing the problem fail. Third, we need better classification of the anomaly reports to accurately identify the true numbers of software vs. nonsoftware-related anomalies. This could be accomplished through mission staff training or through mission requirements for recording anomalies.

As with any bug tracking database, the central question is how to leverage the data to the benefit of the project team. We are exploring natural language processing techniques for triaging new anomaly reports according to risk rating and identifying potential root causes based on the anomaly descriptions, similar to those used in previous NASA datasets [1][2].

\section{ACKNOWLEDGMENT}

The work is sponsored by the NASA Office of Safety and Mission Assurance (OSMA) as part of the Software Assurance Research Program (SARP), grant \#NNX08AZ60G.

\section{REFERENCES}

[1] [1] T. Menzies and A. Marcus, "Automated severity assessment of software defect reports," in 2008 IEEE International Conference on Software Maintenance, 2008, pp. 346-355.

[2] [2] T. Menzies, R. Lutz, and C. Mikulski, "Better analysis of defect data at NASA," in Proceedings of Fifteenth International Conference on Software Engineering and Knowledge Engineering, 2003. 